\title{
Universidades e o mercado institucional de alimentos: 0 exemplo da University of British Columbia (UBC), em Vancouver, no Canadá
}

\author{
Estevan Leopoldo de Freitas Coca \\ Universidade Federal de Alfenas - Alfenas - Minas Gerais - Brasil
}

\begin{abstract}
Resumo
Problemas atuais como as mudanças climáticas, a financeirização de bens básicos (ar, água e alimentos, por exemplo) e o paradoxo entre a fome e a obesidade fazem com que uma grande atenção pública seja direcionada aos sistemas agroalimentares. Dentre as diversas ações que têm visado mitigar ou solucionar esses problemas consta a reorientação dos mercados institucionais de alimentos. Nesse contexto, o presente trabalho tem como objetivo discutir políticas alimentares que têm sido implementadas pela University of British Columbia (UBC), em Vancouver, no Canadá, devido ao fato dela possuir dentre os seus principais objetivos institucionais o vínculo entre seu sistema agroalimentar e a sustentabilidade. Como referência teórica é utilizada a abordagem Community-Campus Engagement (Engajamento entre o Campus e a Comunidade - CCE), pois esta possibilita a leitura sistêmica das interações entre as unidades acadêmicas e a população local. Foram utilizados como procedimentos metodológicos a análises de documentos institucionais, a aplicação de entrevista semiestruturadas e a observação participante. Conclui-se que o entendimento da participação da UBC no mercado institucional de alimentos deve levar em consideração a relação dialética entre produção, comercialização e consumo, tendo como base suas interações com a comunidade local.

Palavras-Chave: Mercado institucional. Community-Campus Engagement (CCE). Sistemas agroalimentares locais. UBC. Sustentabilidade.
\end{abstract}

Universities and the institutional food market: the University of British Columbia (UBC) example in Vancouver, Canada

\section{Abstract}

Current problems such as climate change, the financialisation of basic goods (e.g., air, water, and food) and the paradox between hunger and obesity mean that significant public attention is focused on agri-food systems. Among the various actions that have sought to mitigate or solve these problems is the reorientation of institutional food markets. In this context, the present work aims to discuss food policies that have been implemented by the University of British Columbia (UBC) in Vancouver, Canada, due to the fact that it has among 
its main institutional objectives the link between its agrifood system and the sustainability. As a theoretical reference, the Community-Campus Engagement (CCE) approach is used since this allows for a systemic analysis of the interactions between academic institutions and local community. The methodological procedures employed included analysis of institutional documents, application of semi-structured interviews, and participant observation. We conclude that to asses UBC's participation in the institutional food market one must consider the dialectic relationship between production, commercialization, and consumption, based on the institutions' interactions with the local community.

Keywords: Institutional market. Community-Campus Engagement (CCE). Local agri-food systems. University of British Columbia (UBC). Sustainability.

\section{Universidades y el mercado institucional de alimentos: el ejemplo de la University of British Columbia (UBC), en Vancouver, Canadá}

\section{Resumen}

Los problemas actuales como el cambio climático, la financiación de bienes básicos (aire, agua y alimentos, por ejemplo) y la paradoja entre el hambre y la obesidad hacen que una gran atención pública se dirija a los sistemas agroalimentarios. Entre las diversas acciones que tienen por objetivo mitigar o solucionar estos problemas se encuentra la reorientación de los mercados institucionales de alimentos. En este contexto, el presente trabajo tiene como objetivo discutir políticas alimentarias que han sido implementadas por la University of British Columbia (UBC), en Vancouver, Canadá, debido a que posee entre sus principales objetivos institucionales el vínculo entre su sistema agroalimentario y la sostenibilidad. Como referencia teórica se utiliza el enfoque Community-Campus Engagement (Compromiso entre el Campus y la Comunidad - CCE), pues ésta posibilita la lectura sistémica de las interacciones entre las unidades académicas y la población local. Se utilizaron como procedimientos metodológicos para análisis de documentos institucionales, la aplicación de entrevistas semiestructuradas y la observación participante. Se concluye que el entendimiento de la participación de la UBC en el mercado institucional de alimentos debe tener en cuenta la relación dialéctica entre producción, comercialización y consumo, teniendo como base sus interacciones con la comunidad local.

Palabras-Clave: Mercado Institucional. Community-Campus Engagement (CCE). Sistemas agroalimentarios locales. UBC. Sostenibilidad.

\section{Introdução}

Atualmente, diversos acadêmicos, governos, movimentos sociais e instituições dos mais variados tipos têm chamado atenção para a confluência entre as crises climática, energética, financeira e alimentar, processo que vem acompanhado de fatores como a corrida mundial por terras, o paradoxo entre a fome e a obesidade, o aumento das migrações do campo para a cidade e outros (HOLT GIMÉNEZ; SHATTUCK, 2011; SCHNEIDER; MCMICHAEL, 2010). Como parte das reações a esse contexto se destaca a produção/fortalecimento de sistemas agroalimentares locais dotados de maior preocupação com a sustentabilidade social, econômica e ambiental (SAGE, 2012; TILMAN; CLARK, 2014) ${ }^{1}$. São exemplos de ações com essa perspectiva as hortas urbanas (BAKER, 2010; COLASANTI; HAMM;

\footnotetext{
${ }^{1}$ A leitura do conceito de sustentabilidade presente nesse trabalho vem ao encontro da abordagem de Sage (2012), para quem os sistemas agroalimentares, em todos os seus estágios (regimes de propriedade de terra, seleção de sementes, produção, transporte, comercialização, consumo e descarte), possuem relação com o meio-ambiente. Portanto, somente uma análise que considere
} 
LITJENS, 2012; ROMAN-ALCALÁ, 2015a), os conselhos de políticas alimentares (KOC et al., 2008; WEKERLE, 2004; WELSH; MaCRAE, 1998), os mercados de produtores orgânicos locais (VOGT; KAISER, 2008; WITTMAN; BECKIE; HERGESHEIMER, 2012) e o que é mais importante para efeito desse artigo, as políticas de fomento do mercado institucional de alimentos produzidos na escala local (FRIEDMANN, 2007; GRISA et al., 2011; STROHBEHN; GREGOIRE, 2008; VIVERO-POL, 2017). Sobre esse último exemplo, o poder de compra de órgãos públicos como universidades, escolas, hospitais e presídios passa a ser visto como um vetor da promoção de alternativas ao modelo hegemônico de organização dos mercados, o qual está centrado em elementos como a exploração do trabalho, a busca pela obtenção de lucros ampliados e a desconexão entre produção e consumo. Em alguns casos também compõem o mercado institucional estratégias de produção e comercialização de produtos.

Nessas circunstâncias, o presente trabalho tem por intuito discutir a relação da University of British Columbia (UBC), em Vancouver, no Canadá com o mercado institucional de alimentos. Para isso, utiliza-se a abordagem Community-Campus Exchange (Trocas entre o Campus e a Comunidade - CCE). Essa base teórica permite a interpretação da relação sistêmica entre as ações desenvolvidas nas unidades acadêmicas e a população local. Nos últimos anos, a UBC tem adotado, dentre seus objetivos institucionais, a busca pela relação entre a produção e o consumo de alimentos e a sustentabilidade. Isso se dá, dentre outros fatores, em razão de a UBC ser parte integrante do movimento Farm to Cafeteria Canada (Da Fazenda para a Lanchonete Canadá - F2CC), que possui como objetivo modificar a relação que instituições públicas canadenses como escolas, universidades e hospitais possuem com o alimento, de modo a valorizar ações como o maior consumo de produtos locais em suas lanchonetes e restaurantes, a implementação de hortas e o food literacy (educação alimentar) (F2CC, 2011, 2012). O F2CC é um exemplo de medidas adotadas pela sociedade civil do Hemisfério Norte com o intuito de resolver problemas que estão atrelados à inoperância do Estado num contexto de capitalismo neoliberal, nesse caso, por meio do sistema agroalimentar (COCA, 2016). Por mais que muitas desses medidas não possuam nítidos contornos de classe, nem tenham por intuito gerar modificações estruturais nas relações de poder (ALLEN; GUTHMAN, 2006), aqui adota-se a leitura de que elas possuem caráter alternativo pelo fato de serem produzidas por relações sociais que funcionam de modo diverso do modelo hegemônico de organização do processo de alimentação da população como parte da globalização neoliberal (KLOPPENBURG; HASSANEIN, 2006).

O trabalho está organizado em três partes, além dessa introdução e das considerações finais. Primeiramente, são discutidos os procedimentos metodológicos que balizaram o estudo das compras públicas de alimentos pela UBC. $\mathrm{Na}$ sequência, considerando um contexto de crise de identidade por parte das universidades, é feita uma discussão sobre as compras públicas de alimentos com base na abordagem CCE. Por fim, são apresentados resultados que pontuam que as políticas de fomento do mercado institucional de alimentos por parte da UBC podem ser tomadas como um exemplo de manifestações de resistência na escala local contra os efeitos nocivos relacionados ao modelo hegemônico de organização dos

todas essas etapas dimensões como integradas é capaz de contribuir para a produção de processos alimentares de sustentabilidade. 
sistemas agroalimentares, de modo que promovem a integração sistêmica entre a comunidade acadêmica e a população local.

\section{Procedimentos metodológicos}

A pesquisa que deu origem a esse trabalho se desenvolveu entre novembro de 2014 e outubro de 2015 quando o autor realizou um estágio de doutoramento na UBC com a intenção de estudar a possível contribuição do F2CC para a soberania alimentar, a qual, conforme proposta pela coalizão global de movimento rurais La Via Campesina, foi entendida como o direito de cada povo controlar seu processo de alimentação (COCA, 2016). Por ser considerada como um dos exemplos mais emblemáticos de instituição pública envolvida com o F2CC na província de Bristhi Columbia (Colúmbia Britânica), a UBC foi escolhida para ser parte da pesquisa.

Assim, num primeiro momento, foram pesquisados materiais escritos que pudessem dar base teórica para a pesquisa empírica. Esses materiais se dividiram em trabalhos acadêmicos e documentos institucionais. Os primeiros foram analisados por meio do sistema integrado de bibliotecas da própria UBC, o qual possibilita o acesso a materiais impressos e digitais (inclusos os principais periódicos dedicados a discussão da Economia Política do alimento no mundo). Foram pesquisados centenas de livros, teses, dissertações e artigos de periódicos que abordassem de modo direto ou indireto os seguintes temas: modelo CCE, sistemas agroalimentares locais, regimes agroalimentares globais, soberania alimentar e mercado institucional de alimentos. O segundos foram pesquisados nas Webpages da UBC e do F2CC com o intuito de compreender a abordagem que essa instituição universitária possui do seu próprio sistema agroalimentar. Ambos tipos de materiais (acadêmicos e institucionais) foram organizados em pastas que remetiam aos temas centrais supracitados no software de referenciamento bibliográfico Mendeley para posterior consulta.

Aproveitando o contexto de estágio realizado na própria UBC, o autor do trabalho também utilizou-se da observação participante para atingir os objetivos propostos. Seguindo as orientações de Foote-White (2004) foram tomadas medidas como: negociação com os sujeitos estudados para inserção do pesquisador nas dinâmicas em questão, anotação dos principais processos observados numa caderneta de campo, reconhecimento da condição de outsider do pesquisador e da complexidade que caracteriza as relações sociais. Isso se deu por meio do envolvimento do pesquisador em atividades como as seguintes: diversos tipos de trabalhos de pesquisa e extensão desenvolvidos pela fazenda experimental da UBC, rede de comércio solidário de alimentos desenvolvidos por membros da comunidade acadêmica seguindo o modelo de Community Supported Agriculture (Agricultura Apoiada pela Comunidade) e participação em eventos organizados pela Faculty of Land and Food Systems (Faculdade da Terra e Sistemas Alimentares) da UBC com o intutio de debater, divulgar e celebrar a produção local de alimentos.

Ressalta-se também a realização de 09 entrevistas semiestruturadas com o intuito de produzir informações densas sobre a realidade em questão (POPE et al., 2000; TURRA NETO, 2012). Os sujeitos entrevistados se dividiram nos seguintes grupos: membros do $\mathrm{F} 2 \mathrm{CC}$, gestores da fazenda experimental da UBC e food advocates (defensores dos alimentos) que atuam na província de British Columbia. 
Essas entrevistas foram analisadas por meio do software Atlas.ti. Apesar dos 09 entrevistados terem oferecido importantes contribuições diretas ou indiretas para o entendimento do processo estudado, para efeito desse trabalho destacou-se a contribuição da Coordenadora de Comunicação e Extensão do Centro de Sistemas Alimentares Sustentáveis da UBC Farm, pelo fato de possuir uma leitura ampla das políticas alimentares dessa instituição universitária.

\section{As compras institucionais de alimentos e a abordagem CCE}

A discussão sobre a contribuição das universidades para a promoção de sistemas agroalimentares justos por meio do mercado institucional deve partir da definição do papel social que elas exercem nas comunidades onde estão inseridas (SIERRA YOUTH COALITION, 2014; VOGT; KAISER, 2008). De acordo com Santos (1989), desde a década de 1960, as instituições universitárias se veem frente a três crises que impedem uma leitura clara a respeito da sua identidade, o que inclui sua função social. A primeira é de hegemonia, pois a universidade - especialmente a de caráter público - perde a capacidade de ofertar à sociedade soluções que até outrora Ihe eram outorgadas. Em parte, isso se explica pelo fato de que as universidades se tornaram ainda mais funcionais ao mercado capitalista de trabalho, tendo como um dos seus principais objetivos a formação de mão-de-obra para as empresas. A segunda crise é de legitimidade, pois as universidades passaram a ser vistas como representantes da alta cultura, a qual é inacessível para a totalidade da população, especialmente os mais pobres. Nesse caso, o conhecimento produzido nas unidades acadêmicas é lido como uma ferramenta para a manutenção do status quo. A terceira crise, de cunho institucional, se alicerça nas duas citadas anteriormente. Sua principal característica é a confusão entre os modelos de gestão empresariais com os objetivos da atividade acadêmica frente aos principais problemas do mundo contemporâneo. Como exemplo disso pode ser citado o fato de que os critérios de avaliação da atividade docente se tornaram mais quantitativos, especialmente no que se refere ao número de publicações, do que qualitativos.

Nesse sentido, pensar a superação da crise que caracteriza as universidades no mundo contemporâneo exige, primeiramente, redireciona-las ao envolvimento direto com algumas das questões sociais, ambientais, políticas, econômicas e culturais que são mais preementes de solução. Para que isso ocorra, a universidade precisa ser vista como um elemento que não é atingido de modo direto pelo processo de mercantilização dos bens comuns.

No que se refere aos sistemas agroalimentares, percebe-se, por meio de uma remodelagem das políticas relacionadas ao mercado institucional uma possibilidade de ao menos parcialmente contribuir para a superação dessa crise de identidade das universidadades. Têm sido crescente o número de ações adotadas por unidades acadêmicas que, influenciada por food advocates, visam desenvolver políticas alimentares alternativas, rompendo com tem sido pontuado como neoliberal diet (dieta neoliberal) (COCA; BARBOSA JUNIOR, 2018; OTERO et al., 2015). Uma referência das discussões que caminham nesse sentido se alicerça na aborgem CCE, que é definida por Levkoe e outros (2016, p. 35, tradução nossa) do seguinte modo:

no nível mais básico, CCE pode ser descrito como uma situação em que atores estabelecidos no campus (incluindo alunos de graduação, bolsistas 
de pós-doutorado, instrutores, professores e suas instituições) trabalham em parceria com profissionais de diversos setores da comunidade mais ampla (incluindo a comunidade privada, pública e sem fins setores).

Nesses casos, busca-se compreender as demandas conjuntas da sociedade na qual as unidades acadêmicas estão inseridas e após isso, criar estratégias articuladas para sua superação. Isso pode se dar por meio de projetos de ensino, pesquisa e extensão, os quais compõem o tripé das estruturas universitárias. Todavia, os mesmos autores chamam a atenção para o fato de que dentre as abordagens CCE deve-se diferenciar as que possuem caráter convencional das que possuem caráter transformador. As convencionais colocam a universidade como um ente superior, dotado de saberes que devem orientar a comunidade nas tomadas de decisão. Além disso, elas também se caracterizam pelo não envolvimento direto dos membros da comunidade acadêmica com os assuntos que concernem à população local de um modo mais amplo. Isso assume uma perspectiva positivista de separação entre quem pesquisa e quem é pesquisado, quem estuda e quem é estudado, quem propõe e quem apenas é "beneficiado" pela proposição (TRIVIÑOS, 2009). Um exemplo de crítica contundente a esse modelo pode ser encontrada no trabalho de Bortolin (2011), o qual mostra como nas atividades conjuntas entre a universidade e comunidade, a primeira se beneficia de modo mais enfático dos resultados do que a segunda. As transformadoras partem da compreenssão de um conhecimento que é produzido em caráter conjunto entre a universidade e a comunidade, visando romper com as hierarquizações. Pontua-se a importância da leitura de Freire (1970) para o entendimento da capacidade transformadora dos processos educacionais. Assim, existe uma interação dialética entre os entes envolvidos, os quais são entendidos como parte de um todo complexo, sendo iguais em relação à sua importância. Assim, torna-se possível pensar de modo mais efetivo a possibilidade de modificações estruturais que superem as principais contradições sociais, políticas, econômicas, culturais e ambientais. Isso porque, como pontuado por Butcher e outros (2011), tanto os objetivos como os resultados são pensados de modo compartilhado entre centros universitários e população local.

De tal modo, por meio da abordagem CCE, tomada em sua ótica transformadora, é possível se compreender que frente aos já citados problemas que caracterizam os sistemas agroalimentares, ações desenvolvidas nas, pelas e com as universidades pontuam as unidades acadêmicas não apenas como responsáveis pela leitura e interpretação conjuntural, como também pela tomada de posição como parte das soluções pretendidas. É nesse prisma que o mercado institucional passa a ser lido como de grande valia na participação das universidades em estratégias que visam modificar as regras que regem os sistemas agroalimentares na atualidade. Grande parte dos trabalhos que buscam compreender isso focam na capacidade de compra das universidades, a qual é vista como capaz de fomentar a economia local por meio do pagamento de preços justos aos pequenos produtores (De SCHUTTER, 2014; FRIEDMANN, 2007; KNIGHT, 2013). Evidentemente, não se pode negar que através da orientação da sua capacidade de compra para práticas solidárias e sustentáveis, as universidades podem fornecem grande contribuição para democratização dos sistemas agroalimentares. Todavia, além disso, defende-se nesse texto também a importância das ações de produção e comercialização que são vinculadas às práticas de ensino e extensão. De tal modo, a universidade participa do 
mercado institucional por meio de uma perspectiva dodata de significativa complexidade.

Para exemplificar essa compreensão, na próxima seção são apresentados alguns resultados de pesquisa realizada na UBC com vistas a comprrender as políticas de produção do seu sistema agroalimentar e consequentemente como ela se relaciona com o mercado institucional de alimentos.

\section{A UBC e o mercado institucional de alimentos}

Dentre os food advocates têm crescido a compreensão de que as unidades acadêmicas podem desempenhar um relevante papel no fortalecimento das estratégias de de fomento do mercado institucional de alimentos (COCA, 2016; LEVKOE et al., 2016; ROMAN-ALCALÁ, 2015b; WILKINS, 2005). Tal leitura é sustentada pelo fato de que esses espaços de produção do conhecimento possuem um elevado poder de compra, além de desenvolverem atividades de pesquisa e extensão que podem contribuir com o desenvolvimento da agricultura e da segurança alimentar em nível local (SIERRA YOUTH COALITION, 2014). Nesse sentido, com base na abordagem CCE de característica transformadora, as universidades são vistas como parte integrante da busca por soluções para a mitigação ou superação de alguns dos mais proeminentes problemas que acometem os sistemas agroalimentares em suas diversas escalas e processos, todavia, especialmente no que se refere à comunidade local. Nesse caso, o entendimento não é que a universidade seja uma parte isolada de onde podem emergir respostas técnicas ou práticas para os principais problemas que acometem a comunidade, mas como parte de um sistema que articula as instituições acadêmicas e a população local por meio de uma totalidade.

Isso pode ser percebido na UBC, campus de Vancouver ${ }^{2}$, onde parte da produção da UBC Farm (fazenda experimental) é vendida para unidades de comercialização de alimentos que funcionam no campus e também para o UBC Hospital (hospital universitário). Devido à diversidade dessas ações, a UBC integra a rede $\mathrm{F} 2 \mathrm{CC}$ - a qual, como já ressaltado, é uma das principais incentivadoras de políticas de fomento do mercado institucional canadense - em duas das suas principais modalidades: o Farm to Campus (Da Fazenda para o Campus) e o Farm to Hospital (Da Fazenda para o Hospital).

O campus de Vancouver da UBC possui 44.442 estudantes de graduação, 9.984 estudantes de pós-graduação, 4.975 professores e 9.959 funcionários. Assim, a comunidade acadêmica é formada por 65.658 pessoas (UBC, 2018). Dentre esses, uma parcela significativa, especialmente os estudantes, faz grande parte das suas refeições no próprio campus. A maior parcela das políticas de alimentação dessa unidade acadêmica são gerenciados pelo UBC Food Services (Serviços de Alimentação da UBC). Ele é responsável por mais de 9.000 refeições que são servidas diariamente nas residências acadêmicas, onde vivem mais de 4.500 estudantes; em pontos de venda como cafés, bistrôs, restaurantes, food trucks (comidas vendidas em caminhões) e também em ocasiões especiais como coquetéis para eventos científicos, reunióes acadêmicas e outros (UBC, 2015b). Assim, somente o fato de a

\footnotetext{
2 Além do campus de Vancouver, a UBC possui o campus de Okanagan, o qual conta com 8.300 estudantes. Todavia, as análises contidas nesse trabalho se referem explicitamente ao campus de Vancouver.
} 
UBC possuir um setor que possui como responsabilidade exclusiva pensar as dinâmicas alimentares que se dão em seus centros universitários já indica a importância do presente trabalho.

No que se refere às compras de alimentos, a UBC possui algumas aberturas regimentais que lhe permitem adquirir produtos locais, o que tem como objetivo direto fortalecer seu envolvimento com a comunidade na qual está inserida. Tal ação, como apresentado anteriormente, é um dos pilares da abordagem CCE. O documento UBC's Sustainability Academic Strategy (Estratégia de Sustentabilidade Acadêmica da UBC), por exemplo, ao mesmo tempo em que pontua a sustenbilidade como um objetivo para os diversos tipos de atividades que são desenvolvidas no interior dos campi dessa universidade, também destaca como a produção, a compra e a comercialização de alimentos podem contribuir para isso (UBC, 2009). De maneira mais específica, o documento UBC Sustainable Campus Food Guide (Campus Sustentável Guia Alimentar da UBC) traz orientações para que estudantes, professores e funcionários contribuam para que a UBC produza um sistema agroalimentar que preencha as necessidades da presente geração sem comprometer as gerações futuras. São destacadas várias medidas que podem ser adotadas para atingir esse objetivo como a diminuição do consumo de carne, o aumento do consumo de produtos orgânicos, a preferência pela aquisição de produtos que tenham o selo de Fair Trade (Comércio Justo) e o estabelecimento da meta de fazer com que a UBC se torne um líder na adoção de práticas sustentáveis de compra de alimentos (UBC, 2013).

Vale ressaltar ainda que cerca de $53 \%$ dos alimentos que são adquiridos pelo UBC Food Services é cultivado ou processado a pelo menos $250 \mathrm{~km}$ de distância, além de que na última década houve uma aumento de $100 \%$ na aquisição de maçãs orgânicas, ovos oriundos de galinhas criadas fora de gaiolas e aves locais (UBC, 2014). Isso contribuiu para que em 2012, a UBC fosse a primeira universidade a receber 0 prêmio Golden Carrot (Cenoura de Ouro), que é oferecido pela rede $F_{2} C C$, reconhecendo sua excelência na promoção de ações de Farm to Cafeteria.

Portanto, para efeito da discussão aqui apresentada, o elemento mais marcante da atuação da UBC no mercado institucional é a compra de alimentos.Um dos objetivos de suas políticas alimentares é contribuir com a produção local. Todavia, ela também atua de modo direto em outras etapas dos sistemas agroalimentares como é o caso da produção e da comercialização. Evidentemente, essas atividades não são pensadas como fins em si mesmas, mas como parte de ações de ensino, pesquisa e extensão.

Um elemento central na promoção de ações de Farm to Cafeteria na UBC, campus de Vancouver, é a UBC Farm, que é integrada à Faculty of Land and Food Sytems e administrada pelo Centre for Sustainable Food Systems (Centro para Sistemas Alimentares Sustentáveis). Ela possui 24 ha, localizados no sul do campus universitário (Figura 01).

Figura 1 - Localização da UBC Farm 


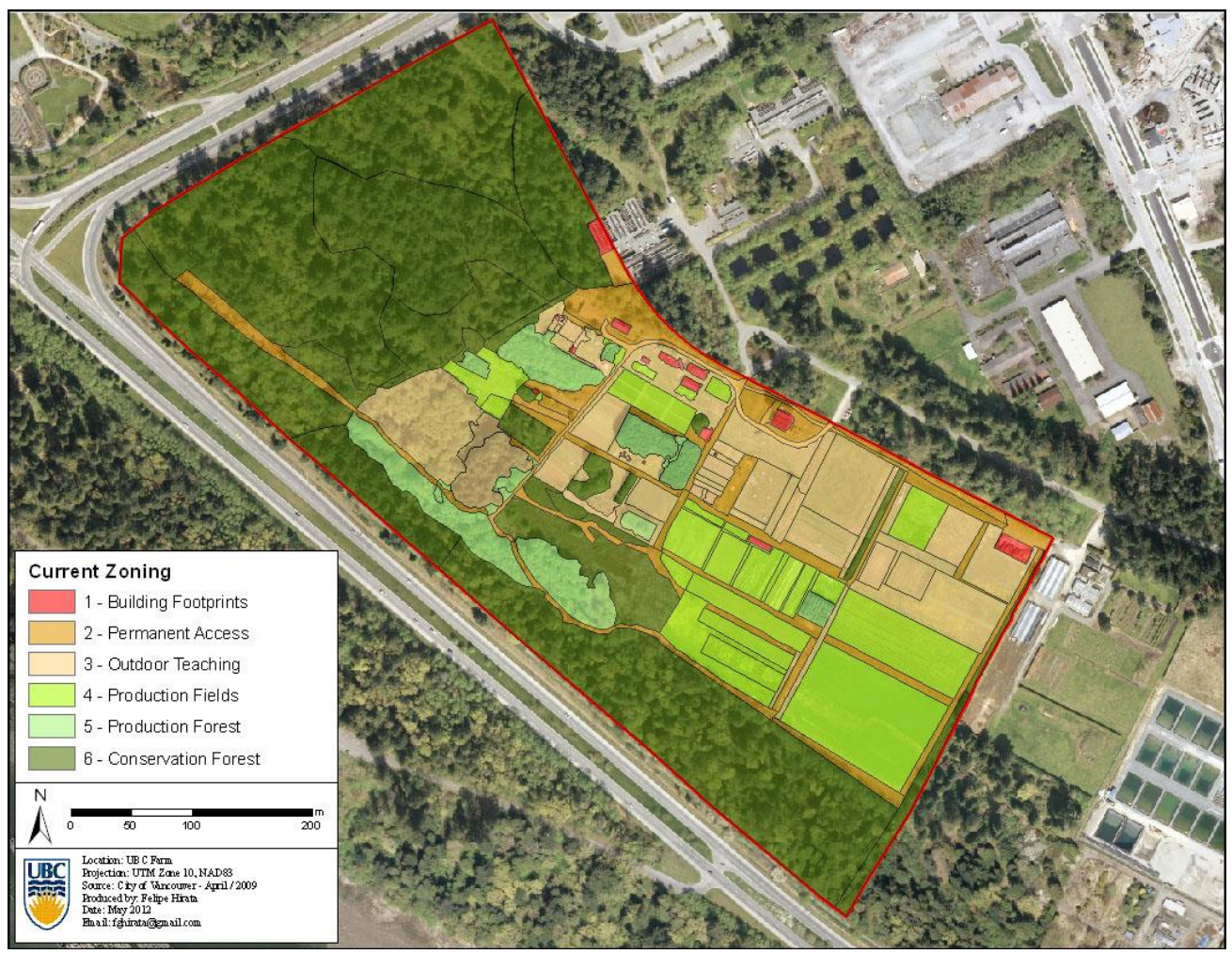

Fonte: http://cfs2.sites.olt.ubc.ca/files/2012/07/UBC_FARM_FH_Current_20120517th.jpg

Na UBC Farm são cultivadas mais de 200 espécies de frutas, vegetais e ervas, além de experiências com a produção de mel e ovos e a criação de animais no sistema de pasto aberto. Essa fazenda experimental é considerada como totalmente orgânica, pois desenvolve sua produção de acordo com os requisitos da British Columbia Certified Organic Management Standards (Normas de Certificação de Gestão Orgânica da Colúmbia Britânica) e da North Okanagon Organic Association (Associação Orgânica de Norte Okanagon), além de ser fiscalizada anualmente por um Environmental Health Officer (Oficial de Saúde Ambiental). Devido a isso, são adotadas práticas como a convivência com alguns insetos considerados benéficos, a retirada manual de ervas daninhas e outros. Na Figura 02 constam algumas imagens da UBC Farm. 


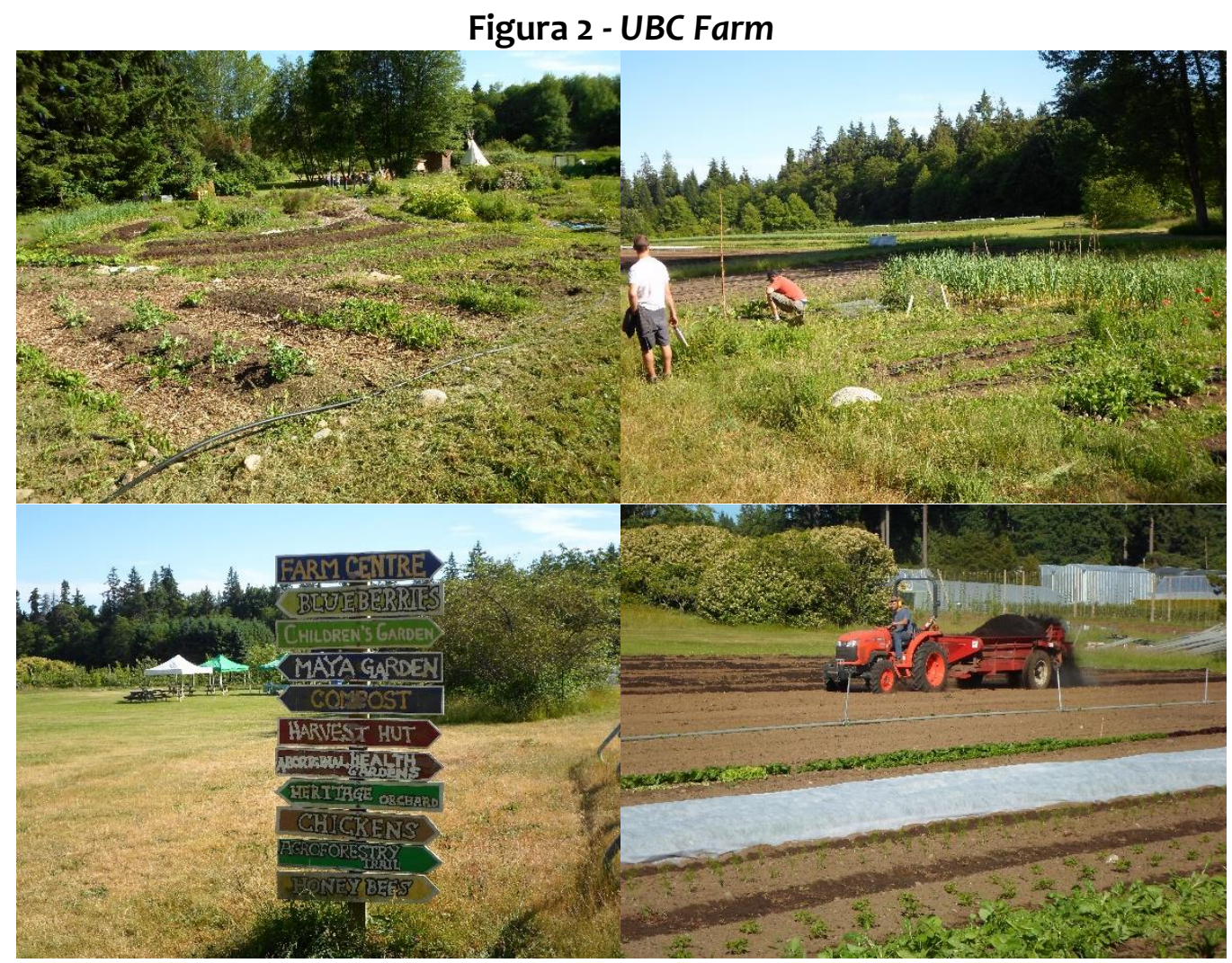

Fotografia: Autor - Junho de 2015.

Os principais canais de comercialização da UBC Farm são: i) três feiras realizadas aos sábados e às terças na própria UBC Farm e às quartas, ao lado da UBC Bookstore (Livraria da UBC). Essas feiras não ocorrem o ano todo no mesmo formato, pois têm suas estruturas adaptadas de acordo com as estações; ii) sistema de Community Support Agriculture (Agricultura Apoiada pela Comunidade - CSA), em que pessoas físicas - da comunidade acadêmica ou não - pagam uma taxa de $C \$ 550$ para receber uma vez por semana, durante vinte semanas consecutivas (verão e outono), uma cesta com produtos cultivados através de métodos orgânicos pela UBC Farm e; iii) vendas de atacado, incluindo ações de Farm to Cafeteria.

Desde 2003, a UBC Farm tem se inserido em atividades de Farm to Cafeteria, vendendo parte de sua produção para unidades de comercialização de alimentos localizadas no próprio campus da UBC, em Vancouver. O primeiro cliente foi a rede de restaurantes Sage Bistrot, especializada em comidas finas (estilo gourmet). Nesse primeiro ano foram comercializados cerca de $C \$ 1.100$ em variados tipos de verduras e legumes. Ou seja, a UBC Farm inseriu-se em ações de Farm to Cafeteria, primeiramente, através da venda de produtos alto valor, destinados a um limitado nicho de consumidores (UBC, 2014). Isso se deu porque, nesse período, a produção da UBC Farm ainda era pequena, limitando-se a poucos produtos de alto valor comercial que "[...] só poderiam ser comprados por esse restaurante muito chique" (S. L. Coordenadora de Comunicação e Extensão do Centro de Sistema agroalimentares Sustentáveis da UBC Farm - Vancouver - 16/06/2015). Em 2006, em outra ação pontual, a UBC Farm também passou a fornecer beterraba e abóbora para elaboração de pizzas pela lanchonete Pie R Squared, que funciona no Union Student Building (Prédio da União Estudantil). 
Todavia, foi no ano de 2007 que a inserção da UBC Farm em ações de Farm to Cafeteria se tornou mais intensa, tornado-se uma referência para outras instituições do tipo no Canadá. Nesse ano, o chefe de cozinha Steve Golob, que era encarregado das refeições servidas no Vanier Hall (conjuntos de residências estudantis), estabeleceu uma parceria com a UBC Farm para que as refeições ali preparadas fossem compostas predominantemente por alimentos saudáveis e de origem local. Com isso, os cerca de 2.500 estudantes que se alimentavam no Vanier Hall diariamente, pagando cerca de $C \$ 5,90$ por refeição, passaram a contribuir com a manutenção e expansão da UBC Farm.

\begin{abstract}
Esse chefe queria tornar as refeições mais locais e com o gosto melhor, por isso, ele passou a buscar descobrir se ele poderia obter o mesmo tipo de produto que o Sage Bistrot estava usando, porque ele pensou que se eles tinham estas cenouras realmente agradáveis, que eram laranjas e roxas, ele também queria. Mas ele foi informado de que, basicamente, a UBC Farm não poderia fornecer, porque nós ainda não tínhamos esse nível de produção. Mas, mesmo assim, ele telefonou para a UBC Farm e, em seguida, o gerente de produção, disse: "Oh bem, não podemos dar-lhe tudo o que quer, mas nós vamos dar-lhe um pouco". E assim, o relacionamento começou lentamente e em pequena escala (S. L. Coordenadora de Comunicação e Extensão do Centro de Sistema agroalimentares Sustentáveis da UBC Farm - Vancouver - 16/06/2015).
\end{abstract}

Dali em diante, a UBC Farm passou por um processo de reestruturação para poder ampliar sua escala de produção afim de atender não só os projetos de Farm to Cafeteria, como também outros canais de comercialização. Ou seja, sua relação com o mercado institucional de alimentos se tornou mais ampla e complexa. Dentre essas mudanças, destacam-se a priorização de outros tipos de produtos, além dos que eram destinados a nichos de mercado e a aquisição de refrigeradores para poder comercializar também em períodos de entressafra. Com isso, atualmente, somente em vendas realizadas junto ao UBC Food Services, a UBC Farm obtém anualmente C\$ 25,000. Compõem seu hall de clientes 9 restaurantes ou lanchonetes localizados no campus e 15 fora dele. Evidentemente, o dinheiro obtido em tais ações é utilizado com gastos operacionais da fazenda experimental, pois ela não é uma empresa como outra qualquer.

De tal modo, a relação da UBC Farm com projetos de Farm to Cafeteria, primeiramente, se deu através de ações de Farm to Campus, que é centralizado no ensino, produção e consumo de alimentos por parte das instituições acadêmicas (F2CC, 2012b). Contudo, atualmente, ela também tem buscado inserir-se em estratégias para ampliar as vendas para o UBC Hospital, o qual é administrado pela Vancouver Coastal Health, possuindo, dentre seus números cerca de 800 funcionários e mais de 21.000 atendimentos por ano (VANCOUVER COASTAL HEALTH, 2015).

[...] ultimamente, estamos focados mais especificamente na exploração agrícola para o Hospital, que é um subconjunto do movimento Farm to Institution, e a razão para isso é que, pensamos que como um projeto específico da UBC, existe um relativo sucesso do Farm to Cafeteria nas residências universitárias, porém, o Farm to Hospital tem obtido pouco sucesso, por isso, agora, nós estamos centrando nossos esforços nisso (S. L. Coordenadora de Comunicação e Extensão do Centro de Sistemas Alimentares Sustentáveis da UBC Farm - Vancouver - 16/06/2015). 
O consumo de alimentos do UBC Hospital é gerenciado pela empresa multinacional Sodexo, que possui como principal fonte de aquisição de produtos a estadunidense Gordon Food Services. Através do projeto-piloto Farm to Healthcare, financiado pelo banco Vancity, ficou estabelecido que durante o biênio 2015-2016 esse distribuidor daria preferência aos produtos cultivados pela UBC Farm, como forma de incrementar o sistema agroalimentar universitário (SINE et al., 2014).

Dentre as principais motivações para as ações de Farm to Hospital está a contribuição que uma alimentação saudável pode oferecer para o processo de recuperação daqueles que estão passando por um período de tratamento médico em unidades de saúde. Isso pode ser percebido no exemplo citado pela Coordenadora de Comunicação e Extensão do Centro de Sistemas Alimentares Sustentáveis da UBC Farm.

[...] eu recebi uma carta de uma pessoa do norte da Colúmbia Britânica que ficou no hospital por um longo tempo e, basicamente, disse que a parte mais difícil de estar num hospital é a comida, porque era muito deprimente, era uma ideia de comida que não era comida, era tudo congelado, e era preciso apenas esquentá-la no micro-ondas para você comê-lo e a qualidade é muito desagradável (S. L. Coordenadora de Comunicação e Extensão do Centro de Sistemas Alimentares Sustentáveis da UBC Farm Vancouver - 16/06/2015).

Contudo, vale ressaltar que no caso da comercialização com hospitais existe um desafio a mais, "[...] porque as normas de segurança alimentar são mais rigorosas. Geralmente, alguns hospitais têm orçamentos mais baixos e por isso, a prestação de serviços de alimentação é bem menos flexível" (S. L. Coordenadora de Comunicação e Extensão do Centro de Sistemas Alimentares Sustentáveis da UBC Farm - Vancouver - 16/06/2015). Isso se dá porque, no Canadá os hospitais adotam a Hazard Analysis Critical Control Point (Ponto de Controle e Análise Crítica de Riscos - HACCP), que é recomendada pelo Codex Alimentarius Commission (Comissão de Códigos Alimentares), da Organização das Nações Unidas (ONU). Esse conjunto de normas refere-se não só ao produto final, mas também ao seu processo de produção. Atender ao HACCP tem sido o grande obstáculo para que a UBC Farm se consolide como um importante fornecedor de alimentos para o UBC Hospital (SINE et al., 2014).

Assim, as ações de Farm to Cafeteria que têm sido desenvolvidas no campus de Vancouver da UBC possuem como elemento central a UBC Farm, que apesar de ter diversas estratégias de inserção no mercado, na verdade, é um espaço de produção do conhecimento, ou seja, sua função primária não é atuar como empresa. Devido a isso, dentre as motivações que estão por trás dessas iniciativas destacam-se a criação de novas oportunidades de mercado para agricultores locais, o apoio a economia local e a oferta de alimentos de qualidade para pessoas que são atendidas por instituições públicas.

[...] nós somos um centro agrícola de pesquisas em sistemas alimentares e temos um programa de formação de agricultores. Vemos o Farm to Institution como uma espécie de elo perdido no sistema agroalimentar, porque as instituições são financiadas com dinheiro público, de modo a que o dinheiro local e dinheiro do contribuinte local devem apoiar também as economias locais. [...] Então, a UBC Farm gostaria de ver a comida institucional tornar-se mais socialmente justa, por exemplo, as pessoas que 
estão em escolas ou hospitais ou até mesmo prisões merecem se alimentar com comida local e de alta qualidade como qualquer outra pessoa [...] (S. L. Coordenadora de Comunicação e Extensão do Centro de Sistemas Alimentares Sustentáveis da UBC Farm - Vancouver - 16/06/2015).

De tal modo, essas ações desenvolvidas no campus de Vancouver da UBC são exemplos de como os sistemas alimentares de unidades acadêmicas podem ser utilizados para a expansão de ações de Farm to Cafeteria, problematizando, assim, seu processo de inserção no mercado institucional. Tendo como base a abordagem CCE em sua versão transformadora, percebe-se que através do seu poder de compra e de ações voltadas à produção do conhecimento, a UBC tem participado de modo direto da busca pela mitigação/superação de alguns dos problemas que caracterizam o sistema agroalimentar local.

\section{Considerações finais}

Esse trabalho trouxe algumas observações sobre a participação da UBC, campus de Vancouver no mercado institucional de alimentos. A adoção de ações por parte dessa unidade acadêmica visando relacionar o processo de alimentação com a promoção da sustantabilidade pode ser vista como forma de destacar como no contexto atual, a preocupação com os efeitos nocivos do modelo hegemônico de orgânização dos sistemas agroalimentares em escala global, ganham importância no debate sobre a multidimensionalidade da sustentabilidade e na busca de respostas produzidas na escala local. Essas medidas, apesar de seu alcance pontual, não podem deixar de ser lidas pelo seu caráter alternativo, onde se alia a crítica e a proposição. É por isso, que a discussão aqui apresentada relacionou as políticas alimentares da UBC com a abordagem CCE em sua versão transformadora. Em outros termos, foi-se constatado que as estratégias de participação da UBC no mercado institucional de alimentos, mesmo que limitadas em seu escopo, são pensadas dentro de um contexto mais amplo que remete a alguns dos principais problemas que caracterizam o sistema agroalimentar da Columbia Britânica.

Seguindo o objetivo institucional da UBC de aliar a produção e o consumo de alimentos com a sustentabilidade, a UBC Farm concentra as principais iniciativas com caráter alternativo. Essas ações envolvem elementos como atividades de extensão, comércio de alimentos nas cantinas e restaurantes e a oferta de alimentos por meio do UBC Hospital. Isso reforça a leitura de que a universidade é dotada de um sistema agroalimentar próprio, mas que todavia, está vinculado por processos diretos e indiretos a outros sistemas agroalimentares. Também pode-se destacar que esses exemplos denotam que a inserção da UBC, campus de Vancouver no mercado institucional de alimentos não se dá apenas por meio da compra, mas também através de ações que contemplam outras etapas e processos como a produção e a comercialização.

Assim, o exemplo da UBC pode ser destacado como parte de um contexto em que instituições públicas passam a ser vistas como dotadas de oferecer contribuições com caráter multidimensional num contexto de desenvolvimento regional. Em outros termos, a universidade aqui não é entendida apenas como um espaço de construção do conhecimento, mas também como consumidora e propositora de bens e serviços que estão atrelados a dinâmicas mais amplas. 


\section{REFERÊNCIAS}

ALLEN, P.; GUTHMAN, J. From "old school” to "farm-to-school”: neoliberalization from the ground up. Agriculture and Human Values, Dordrecht,v. 23, n. 4, p. 401415, 2006.

BAKER, L. Tending cultural landscapes and food citizenship in Toronto'S community gardens. Geographical Review, New York, v. 94, n. 3, p. 305-325, 2010.

BORTOLIN, K. Serving uurselves: how the discourse on community engagement privileges the university over the community. Michigan Journal of Community Service Learning, Ann Arbor, v. 18, n. 1, p. 49-58, 2011.

BUTCHER, J.; BEZZINA, M.; MORAN, W. Transformational Partnerships : A New Agenda for Higher Education. Innovative Higher Education, Genebra, v. 36, n. 1, p. 29-40, 2011.

COCA, E. L. F. A soberania alimentar através do Estado e da sociedade civil: o Programa de Aquisição de Alimentos (PAA), no Brasil e a rede Farm to Cafeteria Canada (F2CC), no Canadá. 2018. Tese (Doutorado em Geografia). Universidade Estadual Paulista (Unesp), 2016.

COCA, E. L. F.; BARBOSA JUNIOR, R. C. Responding to neoliberal diets: school meal programmes in Brazil and Canada. In: GRAY, A.; HINCH, R. (Eds.). A handbook of food crime: immoral and illegal practices in the food industry and what to do about them. Bristol: Policy Press, 2018. p. 347-364.

COLASANTI, K. J. A.; HAMM, M. W.; LITJENS, C. M. The city as an "agricultural powerhouse"? Perspectives on expanding urban agriculture from Detroit, Michigan. Urban Geography, Manchester, v. 33, n. 3, p. 348-369, 2012.

De SCHUTTER, O. The Power of Procurement. Roma, 2014.

FARM TO CAFETERIA CANADA (F2CC). Farm to Cafeteria Canada Network. What is Farm to Cafeteria Canada? Principal Partner Organizations National Manager. 2011.

FARM TO CAFETERIA CANADA (F2CC). Farm to Cafeteria: BC communitties share paths to success. Vancouver, 2012 a.

FARM TO CAFETERIA CANADA (F2CC). Farm to Campus: UBC Dlgs in. Quebec, 2012b. FOOTE-WHYTE, W. Treinando a observação participante. In: GUIMARÃES, A. Z. (Ed.). Desvendando máscaras sociais. Rio de Janeiro: Francisco Alves, 2004. p. 77-86.

FREIRE, P. Pedagogia do Oprimido. Rio de Janeiro: Paz \& Terra, 1970. 
FRIEDMANN, H. Scaling up : Bringing public institutions and food service corporations into the project for a local , sustainable food system in Ontario. Agriculture and Human Values, Dordrecht, n. 24, p. 389-398, 2007.

GRISA, C. et al. Contribuições do Programa de Aquisição de Alimentos à segurança alimentar e nutricional e à criação de mercados para a agricultura familiar. Agriculturas, Rio de Janeiro, v. 8, n. 3, p. 34-41, 2011.

HARVEY, D. O enigma do capital e as crises do capitalismo. São Paulo: Boitempo, 2011.

HOLT GIMÉNEZ, E.; SHATTUCK, A. Food crises, food regimes and food movements: rumblings of reform or tides of transformation? The Journal of peasant studies, Hague, v. 38, n. 1, p. 109-144, 2011.

KLOPPENBURG, J.; HASSANEIN, N. From old school to reform school? Agriculture and Human Values, Dordrecht, v. 23, n. 4, p. 417-421, 2006.

KNIGHT, A. J. Perceived benefits and barriers to local food procurement in publicly funded institutions community-based social marketing. Journal of Extension, v. 51, n. 5, 2013.

KOC, M. et al. Getting Civil About Food: The Interactions Between Civil Society and the State to Advance Sustainable Food Systems in Canada. Journal of Hunger \& Environmental Nutrition, Washington, v. 3, n. 2-3, p. 122-144, 2008.

LEVKOE, C. Z. et al. Collaboration for Transformation: Community- Campus Engagement for Just and Sustainable Food Systems. Journal of Higher Education Outreach and Engagement, Athens, v. 20, n. 3, p. 32-61, 2016.

MORGAN, K.; SONNINO, R. The school food revolution. Public food and the challenge of sustainable development. London: Earthscan, 2008.

OTERO, G. et al. The neoliberal diet and inequality in the United States. Social Science \& Medicine, Amsterdam, v. 142, n. AUGUST, p. 47-55, 2015.

POPE, C. et al. Analysing qualitative data. BMJ, London, v. 320, n. January, p. 5-7, 2000.

ROMAN-ALCALÁ, A. Broadening the land question in food sovereignty to northern settings: a case study of Occupy the Farm. Globalizations, Helsink, v. 12, n. 4, p. 545558, $2015 a$.

ROMAN-ALCALÁ, A. Concerning the unbearable whiteness of urban farming. Journal of Agriculture, Food Systems, and Community Development, Ithaca, v.5, n. 4, p. 179-181, 2015b.

SAGE, C. Environment and food. New York: Routledge, 2012. 
SANTOS, B. S. Da ideia de universidade à universidade das ideias. Revista Crítica de Ciências Sociais, Coimbra, n. 27-28, p. 11-60, 1989.

SCHNEIDER, M.; MCMICHAEL, P. Deepening, and repairing, the metabolic rift. Journal of Peasant Studies, Hague, v. 37, n. 3, p. 461-484, 2010.

SIERRA YOUTH COALITION. On-campus food systems: production, distribution \& best practices, Otawa, 2014.

SINE, E. et al. LFS 450: final project report for the Farm-to-Healthcare Project. Vancouver, 2014.

STROHBEHN, C.; GREGOIRE, M. Local foods: from farm to college and university foodservice. 2008. Disponível em:

<http://medcontent.metapress.com/index/A65RM03P4874243N.pdf>. Acesso em: 16 nov. 2018.

TILMAN, D.; CLARK, M. Global diets link environmental sustainability and human health. Nature, London, v. 515, n. 7528, p. 518-522, 2014.

TRIVIÑOS, A. N. S. Introdução à pesquisa em Ciências Sociais: a pesquisa qualitativa em Educação - o positivismo, a fenomenologia, m marxismo. São Paulo: Atlas, 2009.

TURRA NETO, N. Pesquisa qualitativa em Geografia. XVII Encontro Nacional de Geógrafos - XVII ENG. Anais...Belo Horizonte: Associação dos Geógrafos Brasileiros (AGB), 2012

UNIVERSITY OF BRITISH COLUMBIA - UBC. Exploring and Exemplifying Sustainability. UBC's Sustainability Academic Strategy. Vancouver, 2009.

UNIVERSITY OF BRITISH COLUMBIA - UBC. UBC Farm: from a student-run initiative to leading a Farm-to-Institution Movement. Vancouver, 2014. Disponível em: <http://ubcfarm.ubc.ca/2014/07/23/from-a-student-run-initiative-to-leading-a-farm-toinstitution-movement/>. Acesso em 16 nov. 2018.

UNIVERSITY OF BRITISH COLUMBIA (UBC). UBC Sustainable Campus Food Guide. Vancouver, 2013.

UNIVERSITY OF BRITISH COLUMBIA (UBC). Scaling up: the evolution of the UBC Dining Hall. 2014. Disponível em: <http://ubcfarm.ubc.ca/2014/01/23/scaling-up-theevolution-of-the-ubc-dining-hall/>. Acesso em 16 nov. 2018.

UNIVERSITY OF BRITISH COLUMBIA (UBC). UBC Food Services. Disponível em: <http://www.food.ubc.ca/>. Acesso em: 12 de nov. de 2015. 
UNIVERSITY OF BRITISH COLUMBIA (UBC). UBC overview \& facts. Disponível em: <https://www.ubc.ca/about/facts.html>. Acesso em: 3 nov. 2015.

VANCOUVER COASTAL HEALTH. UBC Hospital. 2015. Disponível em: <http://www.vch.ca/locations-and-services/find-locations/?site_id=164>. Acesso em: 12 de nov. de 2015.

VIVERO-POL, J. L. The idea of food as commons or commodity in academia. A systematic review of English scholarly texts. Journal of Rural Studies, Aberystwyth, v. 53, n. July, p. 182-201, 2017.

VOGT, R. A.; KAISER, L. L. Still a time to act: a review of institutional marketing of regionally-grown food. Agriculture and Human Values, Dordrecht, v. 25, n. 2, p. 241$255,2008$.

WEKERLE, G. R. Food justice movements: policy, planning, and networks. Journal of Planning Education and Research, Tallahassee, v. 23, n. 4, p. 378-386, 2004.

WELSH, J.; MACRAE, R. Food Citizenship and Community Food Security: Lessons from Toronto, Canada. Canadian Journal of Development Studies/Revue canadienne d'études du développement, Otawa, v. 19, n. 4, p. 237-255, 1998.

WILKINS, J. L. Eating right here: Moving from consumer to food citizen. Agriculture and Human Values, Dordrecht, v. 22, n. 3, p. 269-273, 2005.

WITTMAN, H.; BECKIE, M.; HERGESHEIMER, C. Linking local food systems and the Social Economy? Future roles for farmers' markets in Alberta and British Columbia. Rural Sociology, Basel, v. 77, n. 1, p. 36-61, 2012.

Estevan Leopoldo de Freitas Coca. Professor Adjunto A - Universidade Federal de Alfenas (Unifal-MG).estevan.coca@unifal-mg.edu.br

Como citar: COCA, Estevan Leopoldo de Freitas. Universidades e o mercado institucional de alimentos: o exemplo da University of British Columbia (UBC), em Vancouver, no Canadá. Redes, Santa Cruz do Sul, v. 24, n. 1, p. 45-61, jan. 2019. ISSN 1982-6745. Disponível em: https://doi.org/10.17058/redes.v24i1.12858. 